\title{
Dispositivos lingüísticos de acogida, aprendizaje expansivo e interculturalidad: contribuciones para la inclusión educativa de estudiantes extranjeros ${ }^{1}$
}

\author{
Felipe Jiménez-Vargas ${ }^{2}$ \\ ORCID: 0000-0002-1081-9250 \\ René Valdés Morales ${ }^{3}$ \\ ORCID: 0000-0003-4242-9748 \\ María-Teresa Hernández-Yáñez ${ }^{4}$ \\ ORCID: 0000-0003-2117-2672 \\ Carla Fardella ${ }^{3}$ \\ ORCID: 0000-0001-8936-2435
}

\section{Resumen}

En el marco del aumento progresivo de extranjeros en escuelas públicas en Chile y de los recientes cambios en los patrones migratorios, la presencia de estudiantes sin dominio de la lengua vehicular del sistema educativo se ha incrementado sistemáticamente en los últimos años. En un escenario marcado por la ausencia de políticas educativas que respondan a esta nueva diversidad lingüística, el propósito de nuestra investigación fue comprender cómo las escuelas han respondido a la llegada de estudiantes extranjeros y qué prácticas educativas han implementado para responder a este desafío. Mediante una etnografía escolar de dos años en cuatro escuelas públicas con alta matrícula de extranjeros, se analizaron documentos institucionales, se realizaron observaciones de campo, entrevistas y grupos focales con diferentes actores de las comunidades educativas. A través de un análisis de contenido temático, los resultados revelan la puesta en marcha de diez dispositivos para gestionar las respuestas educativas hacia estudiantes extranjeros. Analizamos tres de estos dispositivos con foco en lo lingüístico a partir de las tensiones y contradicciones que se producen al interior de los centros educativos del cual forman parte, identificando elementos y dimensiones significativas para impulsar aprendizajes expansivos. Discutimos finalmente estos hallazgos a la luz de los avances y desafíos del enfoque intercultural en nuestra región latinoamericana.

\footnotetext{
1-Financiamiento: Proyecto CONICYT/Fondecyt n. 11150831 y Proyecto CONICYT/Fondecyt n. 11190349. Proyecto Anid/Fondecyt n. 3200192.

2- Pontificia Universidad Católica de Valparaíso, Viña del Mar, Chile. Contacto: felipe.jimenez@pucv.cl.

3 - Universidad Andrés Bello, Facultad de Educación y Ciencias Sociales, Valparaíso, Viña del Mar, Chile. Contactos: revalmorales@gmail.com; carla.fardella@unab.cl.

4- Universidad de Santiago de Chile, Santiago, Chile. Contacto: maitehernandezy@gmail.com.
} 


\title{
Palabras clave
}

Inmigración - Interculturalidad - Acogida escolar - Educación pública.

\section{Linguistic devices for welcoming, expansive learning and interculturality: contributions for the educational inclusion of foreign students}

\begin{abstract}
In the context of the progressive increase of foreigners in public schools in Chile and the recent changes in migratory patterns, the presence of students who do not master the working language of the educational system has systematically incremented in recent years. In a scenario marked by the absence of educational policies that respond to this new linguistic diversity, the purpose of our research was to understand how schools have responded to the arrival of foreign students and what educational practices they have implemented to tackle this challenge. Through a two-year school ethnography in four public schools with high enrollment of foreigners, institutional documents were analyzed and field observation, interviews and focus groups were conducted with different actors amidst the educational communities. Through an analysis of thematic content, the results reveal the implementation of ten devices to manage educational responses to foreign students. We have analyzed three of these devices with a focus on linguistics based on the tensions and contradictions that occur within the educational institutions of which they are part, identifying significant elements and dimensions to drive forward expansive learning. Finally, we discuss these findings in light of the advances and challenges of the intercultural approach in our Latin American region.
\end{abstract}

\section{Keywords}

Immigration - Interculturality - School welcoming - Public education. 


\section{Introducción}

\section{La nueva configuración migratoria de la escuela en Chile}

La incorporación de estudiantes extranjeros ha pasado de ser una cualidad circunstancial y secundaria a un fenómeno estructural del sistema educativo chileno. Prueba de ello es una matrícula que se cuadriplica (de 30 mil a 113 mil estudiantes) entre el 2015 y el $2018^{5}$. Adicionalmente, se observa que este aumento progresivo se va concentrando en escuelas de titularidad pública (57\%), a lo que se añade la intensificación del fenómeno en regiones y comunas específicas como respuesta a un patrón de concentración geográfica de los diferentes colectivos migrantes (ELIGE EDUCAR, 2017). Es así como en ciertas escuelas de educación básica, la matrícula de estudiantes extranjeros supera el 60\% de la matrícula total (FUNDACIÓN..., 2017).

A los factores antes señalados debemos anteponer que, como consecuencia de los cambios del patrón en los flujos migratorios de nuestro país, la llegada y consolidación de nuevos colectivos migrantes en el ámbito escolar -la comunidad haitiana y más recientemente refugiados de origen sirio- han generado la aparición de nuevas necesidades educativas (lingüísticas), nuevas barreras escolares (idiomáticas), y por tanto nuevas problemáticas y desafíos para las propias comunidades. Ahora bien y pese al aumento de investigaciones en torno al fenómeno migratorio en nuestro sistema educativo, aumento que hemos podido mapear en una reciente revisión (JIMÉNEZ; VALDÉS; AGUILERA, 2018), a la fecha son prácticamente inexistentes las investigaciones que profundicen en materia de inclusión lingüística. Los trabajos exploratorios de Campos-Bustos (2019) y Sumonte y otros autores (2018) con extranjeros de nacionalidad haitiana permiten avanzar, respectivamente, en la comprensión de los enfoques adoptados intuitivamente por las escuelas para el aprendizaje del español como segunda lengua, así como de la importancia de incorporar instancias de mediación lingüística e incorporar saberes culturales de los colectivos no hispanoparlantes.

Aumento y diversificación migratoria sumado a una incipiente investigación educativa, confluyen en un escenario en el que a la fecha nuestro sistema educativo no ha adoptado formalmente ningún modelo de acogida para favorecer la escolarización de estudiantes extranjeros, lo que podría permitir que ciertas falencias e inequidades se mantengan e incluso sigan actualizándose (RIEDEMANN, 2017). A falta de un modelo, lo que existe, no obstante, son orientaciones y recomendaciones genéricas y no suficientemente articuladas entre sí, que han sido puestas a disposición de las comunidades escolares para ser utilizadas como referentes de gestión de la diversidad. Esto crea una realidad en que las propias escuelas han ido diseñando e implementando de manera autodidacta (POBLETE; GALAZ, 2017), diversos dispositivos para gestionar sus procesos de acogida.

En ese escenario, nos preguntamos de qué forma han enfrentado las escuelas la incorporación masiva de estudiantes extranjeros y qué procedimientos han puesto en marcha para gestionar dicha incorporación. Este artículo busca dar respuesta a estas dos preguntas de investigación, aportando evidencia empírica que permita ahondar en la comprensión

5- Datos obtenidos del Centro de Estudios del Ministerio de Educación. 
del fenómeno migratorio en el ámbito escolar chileno, campo de investigación que ha recibido un significativo interés académico en los últimos años y que se ha traducido por tanto en un notorio aumento de investigaciones en la materia. Lo anterior, en un contexto actual caracterizado por intensos y estimulantes debates en torno a la resignificación de lo intercultural en educación, donde claramente la pregunta por el lugar de los fenómenos migratorios intenta abrirse paso y buscar un espacio en dichos debates.

\section{Migración, escuela e interculturalidad}

Existe amplio consenso en el debate actual en torno a la relación entre interculturalidad y educación en asumir que la educación intercultural latinoamericana ha sido en términos históricos un movimiento, enfoque y propuesta pedagógica dirigidos exclusivamente a minorías indígenas (MONTECINOS, 2004; RIEDEMANN, 2008). En el caso chileno, el programa de educación intercultural bilingüe surgida a mediados de los años 90 pero aún vigente como eje prioritario (CHILE, 2005), sólo contempla como beneficiarios a estudiantes pertenecientes a los nueve pueblos originarios reconocidos por el Estado. Es más, la actualización de la política intercultural en educación (CHILE, 2018b) incorpora un segundo eje de trabajo denominado Interculturalidad para todos y todas que busca incorporar transversalmente en el currículum escolar temáticas y contenidos relativos a los pueblos originarios (CHILE, 2018a). Lo anterior, con el propósito de contribuir al desarrollo de una ciudadanía competente en materia de interculturalidad. Sin embargo, es posible observar que, en estos documentos, la interculturalidad se instala como discurso para favorecer la inclusión de estudiantes extranjeros, pero no se aborda como una línea de trabajo que permita diversificar de forma concreta las prácticas educativas para responder a la diversidad cultural en la escuela y en la sala de clases. Pese a ello, en la última década ha habido un interés exponencial desde el ámbito académico por incorporar la interculturalidad y otros modelos de gestión de la diversidad (JIMÉNEZ, 2014; BRAVO, 2012; MORA, 2018; STEFONE; STANG; RIEDEMANN, 2016) a la comprensión de los fenómenos migratorios en el sistema escolar chileno. Sin embargo, como evidencian los debates latinoamericanos contemporáneos en torno a la educación intercultural (DIEZ, 2004, FERRÃO, 2010; WALSH, 2009) lo intercultural es un significante que está lejos de proponer una interpretación unívoca: más bien predomina una nomenclatura polifónica en base a diferentes definiciones, componentes, principios y propósitos.

Si considerásemos lo anterior como un primer nudo problemático del debate latinoamericano en la materia, el segundo nudo correspondería a la tensión entre su naturaleza pluralista o fragmentada (SÁNCHEZ-MELERO; GIL-JAURENA, 2015) con relación a sus destinatarios. Frente a la pregunta por hacia dónde debiese avanzar la educación intercultural latinoamericana con relación a escolarización de minorías culturales, nuevamente no hay una respuesta categórica: ¿debiese haber una educación intercultural para minorías indígenas por un lado y una educación intercultural para migrantes por el otro?; ¿convendría avanzar hacia una educación intercultural integrada?; en definitiva, ¿qué modelo de interculturalidad permitiría responder de mejor manera al multilingüismo cada día más presente en nuestras aulas? 
Finalmente, y en base a los recientes desarrollos y propuestas del análisis interseccional (CRENSHAW, 2012) en torno a cómo operan e interactúan los procesos de categorización social en la reproducción de las desigualdades (MAGLIANO, 2015), recientes estudios en torno a la escolarización de estudiantes extranjeros (OLMOS; RUBIO, 2014) problematizan el poder explicativo de lo migratorio para entender las desigualdades existentes en los contextos educativos formales.

Si bien el debate en torno a estos tres nudos problemáticos está lejos de ser zanjado, los resultados de nuestra investigación pretenden aportar evidencias empíricas para avanzar en su abordaje conceptual y en sus implicancias prácticas para la experiencia escolar. Retomaremos esto en las discusiones finales.

\section{Dispositivos de acogida al sistema educativo chileno}

Para situar de mejor manera las preguntas de investigación, se hace necesario precisar algunos conceptos centrales. En primer lugar, qué entendemos por dispositivos de acogida; en segundo lugar, qué implica pensar la escuela como un sistema de actividad y los dispositivos de acogida como componentes emergentes de dicho sistema. Finalmente, argumentar cómo estos dispositivos de acogida se pueden constituir en componentes clave del sistema para impulsar aprendizajes expansivos, y de esta manera responder de manera más adecuada a las necesidades de los propios estudiantes, así como de la misma institución escolar.

Articulando diferentes definiciones y contextos de uso (FERNÁNDEZ; GARCÍA CASTAÑO, 2015; GONZÁLEZ-FALCÓN; MOYA; DUSI, 2018; GONZÁLEZ, 2017; OLMOS; LASTRES, 2018; SIMÓ et al., 2014), entendemos como dispositivos de acogida, aquellas medidas y actuaciones educativas creadas institucionalmente, que a través de la activación de recursos personales, didácticos, culturales y metodológicos, son implementadas en las escuelas para organizar las intervenciones educativas orientadas a la inclusión de estudiantes de origen extranjero al sistema escolar. Estos dispositivos de acogida varían en función de diversos criterios: en primer lugar, en función de su finalidad y, por tanto, de las dimensiones educativas implicadas. La evidencia internacional muestra que las principales dimensiones abordadas por las escuelas son la administrativa, la convivencial, la pedagógica y la vinculación con el entorno (ALEGRE; BENITO; GONZÁLEZ, 2012; ESSOMBRA, 2012; GARRETA, 2006; PALAUDÀRIAS; GARRETA, 2008). En el caso de Chile y como hemos podido documentar en investigaciones previas, la mayoría de los dispositivos de acogida se condensan en la dimensión administrativa y en la dimensión convivencial de los procesos educativos (JIMÉNEZ; VALDÉS; AGUILERA, 2018). Otros criterios de variabilidad de los dispositivos son sus destinatarios -profesores, estudiantes y/o familias-, el nivel de formalidad e institucionalización -presente o ausente en los instrumentos de gestión-, el grado de sofisticación y complejidad de los dispositivos, así como el grado de tensión y conflicto que producen a nivel sistémico (2018). 
Felipe JIMÉNEZ-VARGAS; René Valdés MORALES; María-Teresa HERNÁNDEZ-YÁÑ̃EZ; Carla FARDELLA

\section{Los sistemas de actividad: del legado de Vygotsky a los desarrollos de Engeström}

Partimos de la premisa que la escuela es un sistema de actividad. El enfoque histórico-cultural de la actividad se interesa en los procesos de aprendizaje y desarrollo que emergen en contextos institucionalizados de actividades prácticas cultural e históricamente mediadas, enfoque que ha ido cobrando cada vez más influencia internacional en los estudios educativos (YAMAZUMI, 2009). Para comprender esta premisa se hace necesario entonces remitirse a la evolución histórica del enfoque histórico-cultural a través de diferentes generaciones ${ }^{6}$ (ENGESTRÖM, 2009a). Nuestro argumento-guía es que entender la escuela como un sistema de actividad nos permite comprender de mejor manera el lugar que los dispositivos de acogida ocupan al interior de ésta, y la forma en que dichos dispositivos pueden ser utilizados para promover transformaciones expansivas hacia la inclusión plena de estudiantes de origen extranjero.

La primera generación del enfoque histórico-cultural se centra en la figura de Vygotsky y en su propuesta original en torno al concepto de mediación (ERAUSQUIN, 2014). Este modelo mediacional clásico (VYGOTSKY, 1986), indudablemente revolucionario para la psicología dominante de la época y su aplicación al ámbito educativo, (ENGESTRÖM, 2001), tiene no obstante un excesivo énfasis en lo individual en la definición de su unidad de análisis (LARRIPA; ERAUSQUIN, 2008). De esta manera la segunda generación del enfoque histórico-cultural, centrada ahora en la figura de Leontiev, pretende superar las limitaciones del primer modelo otorgando una mayor importancia a la acción colectiva de la actividad por sobre la acción individual, unidad de análisis de la generación vygotskyana (ENGESTRÖM, 2001). Se instala la premisa que la actividad es ante todo una formación colectiva y sistémica (ERAUSQUIN, 2014), que ubica al sistema de actividad como nueva unidad de análisis (ENGESTRÖM, 2009b). Como señala Yamazumi (2009), este cambio muestra que los procesos de desarrollo y aprendizaje son sociohistóricos y ocurren en consecuencia en el contexto de sistemas de actividad culturalmente mediados. Engeström (1987) sintetiza la segunda generación a través de la expansión del triángulo vygotskyano incluyendo tres nuevos elementos: la comunidad que conforma el sistema de actividad, las reglas que regulan sus interacciones, así como la división del trabajo que se establecen entre sus miembros.

Articulando el enfoque histórico-cultural con los de dispositivos de acogida, éstos deben ser pensados como elementos emergentes en la escuela como sistema de actividad, ya sea como nuevos artefactos mediadores del proceso educativo -el caso de las aulas de acogida o los grupos interactivos como veremos-, o bien como nuevos sujetos -los facilitadores lingüísticos o equipos bilingües-. En ambos casos su emergencia ha supuesto para las escuelas el desafío de reconfigurar los roles y estatus de los miembros de la comunidad educativa, la creación de nuevas reglas y procedimientos que guíen las actividades realizadas, así como una nueva coordinación del trabajo realizado por los participantes. Estos cambios y desafíos producen contradicciones internas que sólo

6- Si bien existen tres generaciones de la teoría de la actividad (ENGESTRÖM, 2001), para efectos de esta investigación nos remitiremos sólo al trabajo conceptual desarrollado en torno a las dos primeras. 
podrán ser resueltas, transformando el sistema de actividad mismo (ENGESTRÖM, 2007). Ahora bien, existen diferentes modalidades de transformación de los sistemas de actividad, desde las formas destructivas y regresivas, pasando por las reproductivas hasta las formas expansivas (ENGESTRÖM, 2009b). Para efectos de nuestra investigación importan aquellas en la que la comunidad es capaz de aprender a rediseñar su propia actividad (KONTOS, 2016), esto es, a desarrollar un aprendizaje de naturaleza expansiva.

\section{Aprendizaje expansivo}

El aprendizaje expansivo refiere a los procesos a través de los cuales un sistema de actividad resuelve sus contradicciones internas construyendo e implementando formas cualitativamente diferentes de funcionamiento (ENGESTRÖM 1987, 2007). El aprendizaje expansivo tiene lugar cuando el objeto y propósito de la actividad de un sistema son reconceptualizados, incorporando un horizonte de posibilidades sustancialmente más amplio que en su anterior funcionamiento. Un aprendizaje expansivo supone, a fin de cuentas, un viaje colectivo a través de la zona de desarrollo próximo del sistema de actividad (SANNINO; ENGESTRÖM; LEMOS, 2016).

En términos específicos el aprendizaje expansivo se trata de un proceso cíclico que incluye, idealmente, las siguientes fases (ENGESTRÖM, 2001): 1) cuestionamiento; 2) análisis; 3) modelado; 4) experimentación; 5) reflexión y; 6) consolidación. El proceso expansivo comienza cuando los agentes han podido detectar y discutir las contradicciones internas que se producen en o entre los componentes del sistema, y que han generado el estado de desequilibrio de éste que requiere ser resuelto. El proceso continúa en la medida que los agentes examinan las situaciones problemáticas creadas, rastreando sus orígenes y evolución, por ejemplo, a través de la creación de una imagen de las relaciones sistémicas internas de la actividad (SANNINO; ENGESTRÖM; LEMOS, 2016). La tercera y cuarta fase emergen en tanto los agentes crean y ponen a prueba el nuevo modelo por medio de aplicaciones prácticas. El proceso expansivo culmina en la medida que las soluciones puestas en práctica son analizadas críticamente por los agentes, y si corresponde, enraizadas y apuntaladas como nuevas prácticas y/o elementos del sistema de actividad; lo anterior por ejemplo, a través de la creación de un nuevo artefacto mediador o bien mediante la incorporación de un nuevo agente educativo (ENGESTRÖM, 2009b).

\section{Metodología}

\section{Diseño, enfoque de investigación y participantes}

Desde un diseño cualitativo y un enfoque etnográfico (FLICK, 2014) se realizó una inmersión secuencial y progresiva al campo educativo. Según Díaz de Rada (2013), la etnografía escolar, mediante el análisis de prácticas escolares y el uso de diversas técnicas, facilita la construcción de un diálogo entre la propia experiencia y marcos socioculturales del investigador con las voces y marcos socioculturales de los actores participantes de la investigación. 
Participaron cuatro escuelas públicas: tres de la Región metropolitana y una de la Región de Antofagasta. Participaron estudiantes, apoderados, profesores, asistentes de la educación y equipos directivos. Las cuatro escuelas tenían al momento de participar de la investigación, una matrícula de estudiantes extranjeros superior al 50\%, siendo la nacionalidad haitiana la de mayor aumento porcentual en los últimos años ${ }^{7}$. El trabajo de campo se realizó entre abril de 2017 y septiembre de 2018.

\section{Técnicas de producción y análisis de datos}

Se realizaron 42 entrevistas individuales, 15 grupos focales, 18 observaciones participantes y la revisión de 14 documentos institucionales. Todas estas técnicas se implementaron de modo secuencial. En un primer momento nos insertamos en los espacios formales (como sala de clases) e informales (comedores y espacios de recreación) de las escuelas. En la medida en que emergían dispositivos claves, se realizaron entrevistas individuales y grupos focales con sus actores participantes. Paralelamente, se revisó documentación relevante (proyecto educativo institucional, reglamento interno, plan de gestión de convivencia, protocolos de acogida).

Para el análisis de los datos utilizamos un análisis de contenido categorial (VÁSQUEZ, 1996), que nos permitiera sistematizar y agrupar en categorías los datos textuales, para posteriormente seleccionar aquellas inferencias e interpretaciones que nos permitieran responder nuestras preguntas de investigación. En ese sentido, se establecieron las siguientes etapas de trabajo: 1) lecturas de datos;2) codificación abierta; 3) categorización; 4) triangulación entre técnicas; 5) reunión del equipo de investigación y 6) consenso de los dispositivos relevantes.

\section{Consideraciones éticas}

Para la inmersión etnográfica y la implementación de las diversas técnicas se solicitaron autorizaciones a los directores de escuelas, consentimientos informados a adultos y asentimientos informados a estudiantes, todos ellos visados por el comité de Bioética de la Universidad patrocinante.

\section{Resultados}

\section{Mapeo de dispositivos de acogida}

Los dispositivos etnografiados fueron: 1) protocolo de acogida; 2) fiesta intercultural; 3) pauta de observación con sello intercultural; 4) mediación escolar; 5) grupo diferencial

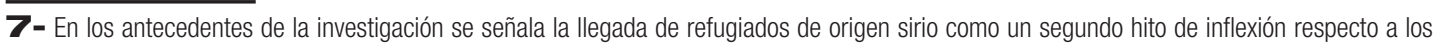
flujos migratorios predominantes hacia Chile. Sin embargo, y pese a nuestro interés, no fue posible incorporar una escuela con estudiantes sirios al trabajo etnográfico. Si bien es posible ofrecer múltiples interpretaciones explicativas de ello, a nuestro juicio la confidencialidad y el anonimato de las familias refugiadas por parte de la administración educativa, fue la principal razón que impidió trabajar con ellas en esta investigación, razón que no sólo aceptamos sino compartimos. 
específıco; 6) estudiantes tutores; 7) cuota migrante; 8) equipo bilingüe; 9) aula de acogida; y 10) grupos interactivos. Aun cuando todos los dispositivos mencionados son utilizados en la actualidad para favorecer la inclusión de extranjeros, no todos ellos surgen necesariamente a partir su llegada. A modo de ejemplo, la mediación escolar surge con anterioridad a la llegada de extranjeros, aunque con el aumento de la migración se haya ampliado notoriamente su uso. Del mismo modo, si bien los diez dispositivos incorporan preferentemente el trabajo con extranjeros, no todos están destinados a cualquier nacionalidad extranjera. A modo de ejemplo, el aula de acogida es un dispositivo reservado para aquellas nacionalidades no castellanohablantes. Para efectos de este trabajo nos centraremos en los tres últimos dispositivos que comparten justamente el propósito de responder a las necesidades lingüísticas y barreras idiomáticas que se instalan en los procesos educativos de estudiantes sin manejo de la lengua vehicular de la escuela, y cuyos destinatarios son precisamente estudiantes de nacionalidad haitiana ${ }^{8}$.

\section{Equipo bilingüe}

Mientras profundizábamos en la comprensión del primer dispositivo implementado por escuelas y que originó nuestra investigación, la creación de protocolos de acogida, supimos de la existencia de una pareja de profesores de nacionalidad haitiana que realizan funciones de apoyo, interpretación y traducción a estudiantes haitianos que no manejan la lengua vehicular. Nos propusimos entender en qué consistía este nuevo dispositivo, consultando a diferentes profesores. Estos explicaron que el equipo bilingüe arriba a la escuela en momentos de incertidumbre y complejidad, pues la brusca matriculación de estudiantes extranjeros implicó a la escuela enfrentarse a una barrera idiomática que limitaba sus posibilidades de hacer efectivos los procesos de enseñanza y aprendizaje.

El propósito general del equipo bilingüe es minimizar al máximo dicha barrera idiomática. En ese sentido, las tareas del equipo se encuadran principalmente en el área técnico-pedagógica teniendo como foco el aula regular. Si bien dentro de sus principales prácticas desarrolladas se encuentran la comunicación con las familias, el diseño de evaluaciones, ofrecer apoyo en el aula de educación especial o clasificar niveles de aprendizaje de los estudiantes, la principal función es dar apoyo lingüístico al interior del aula regular a estudiantes con bajo dominio de la lengua vehicular.

Todas estas acciones se enmarcan en un procedimiento establecido por la escuela y que conjuga una serie de tareas que definen junto a profesores de aula y al equipo de gestión. El trabajo del equipo bilingüe ha sido valorado positivamente por los diversos estamentos de la escuela. De acuerdo a nuestros registros, la razón de esta valoración radica, ante todo, en que su incorporación fue una decisión acertada por parte del director y del equipo directivo, ya que tanto docentes como estudiantes se sienten apoyados en la gestión de la sala de clases.

\footnotetext{
8- Para una revisión completa de los dispositivos restantes recomendamos consultar a Valdés y otros autores (2019).
} 


\section{Aula de acogida}

El aula de acogida corresponde a la creación de un aula específica para el aprendizaje de la lengua vehicular de la escuela. Está a cargo de una profesora de origen haitiano. Es un dispositivo que está destinado a estudiantes que no han estado previamente escolarizados en nuestro sistema educativo y que cuentan en consecuencia con un dominio muy insuficiente del español, situación que les impide seguir con normalidad los procesos educativos en sus respectivas aulas. Por ello, a través del trabajo en pequeños grupos diferenciados por nivel, se realiza un plan individualizado para el aprendizaje de la lectoescritura en español. Tres veces por semana se conforman los grupos para asistir a esta aula y avanzar en el aprendizaje de la lengua. Este modelo plantea una diferencia significativa del trabajo realizado por el equipo bilingüe, ya que como vimos anteriormente, éste supone preferentemente el trabajo en el aula ordinaria. Como hemos documentado a través de conversaciones con diferentes profesores de la escuela que cuenta con esta aula, esta forma de trabajo implica un mayor trabajo individualizado con los estudiantes, pero al mismo tiempo una menor coordinación con los profesores de los cursos a los que pertenecen los estudiantes.

\section{Grupos interactivos}

A diferencia de los dispositivos de acogida que surgen de manera autodidacta en las escuelas, el grupo interactivo corresponde a una de las diferentes actuaciones educativas de éxito que componen el proyecto de comunidades de aprendizaje. A diferencia de los dos dispositivos antes señalados, los grupos interactivos privilegian el aula ordinaria como espacio de aprendizaje, pero con un rediseño metodológico del agrupamiento de los estudiantes a través del aprendizaje colaborativo por estaciones y en grupos heterogéneos de trabajo.

Cuando el proceso de enseñanza-aprendizaje está organizado bajo la lógica de grupos interactivos, el aula está compuesta por diferentes zonas de trabajo y el docente es asistido por un grupo de monitores. Los estudiantes son distribuidos en cada uno de estas zonas de trabajo bajo un principio de heterogeneidad, con lo cual en cada grupo resultante hay mujeres y hombres, estudiantes autóctonos y extranjeros, así como estudiantes con diferentes niveles de dominio del contenido de lo que se va a realizar. Por espacio de veinte minutos, cada grupo acompañado por un monitor realiza una tarea específica (guía de trabajo) que contempla tanto actividades individuales como grupales. El monitor cumple la función de dinamizar las interacciones entre los miembros del grupo, poniendo especial énfasis en aquellos estudiantes con un bajo dominio de la lengua vehicular. Cumplido el tiempo, el grupo se desplaza hacia la siguiente estación de trabajo, y con una nueva actividad y un nuevo monitor resuelve la tarea solicitada. Así por espacio de aproximadamente sesenta minutos. El grupo interactivo favorece, según lo reportado por nuestros informantes, el uso y ejercicio de la lengua vehicular en un contexto natural y multiplicando las oportunidades de interacción entre estudiantes y maximizando el efecto par en los aprendizajes. 


\section{Análisis de dispositivos de acogida: tensiones y contradicciones}

El análisis de los dispositivos de acogida se puede realizar en función de diferentes criterios: 1) de acuerdo a su finalidad, y por tanto, de las dimensiones educativas implicadas -administrativa, pedagógica, convivencial-; 2) sus destinatarios -profesores, estudiantes, familias-; 3) el nivel de formalidad e institucionalización -incorporados o ausentes en los instrumentos de gestión-; 4) el grado de sofisticación y complejidad de los dispositivos; y 5) el grado de tensión y las contradicciones internas que producen en la escuela como sistema de actividad. Como una discusión en torno a los cinco criterios y sus posibles combinaciones queda fuera de los alcances de este trabajo, nos centraremos en los dos últimos, por ser aquellos que presentan un mayor vínculo con la necesidad de impulsar aprendizajes expansivos. Para ello nos centraremos en visibilizar las tensiones, nudos críticos o contradicciones reportadas o documentadas en nuestro trabajo de campo, y de esta manera avanzar hacia una comprensión de cómo estas tensiones podrían traducirse en potenciales oportunidades de transformación y cambio en las escuelas.

Ahora bien, ¿por qué focalizar los análisis en las tensiones y/o contradicciones que emergen de la puesta en marcha de los dispositivos y no en otros criterios posibles? Para responder a ello conviene recordar que un aprendizaje expansivo tiene lugar cuando el objeto o motivo de la actividad de un sistema son reconceptualizados por los miembros de la comunidad con el propósito de incorporar un horizonte de posibilidades sustancialmente más amplio que en el que ofrece el estado actual de la actividad (ENGESTRÖM, 2001); horizonte que se alcanza a través del análisis y cuestionamiento de las propias prácticas, identificando los problemas y contradicciones que supone la introducción de nuevos artefactos, objetos y/o sujetos, y finalmente, creando colectivamente soluciones que están en la zona de desarrollo proximal del sistema de actividad (ENGESTRÖM, 1987). Con el fin de no saturar los análisis con información excesiva, analizaremos solo aquellas contradicciones emergentes en las nuevas dimensiones del sistema de actividad aportadas por Engeström (1987): la comunidad de base, reglas y normas de funcionamiento, así como la división del trabajo.

Con relación a la incorporación de profesores intérpretes que supone nuevos sujetos -y artefactos mediadores- en el sistema de actividad, vemos que es un dispositivo de acogida de alta complejidad que ha generado la aparición de diversas contradicciones, identificando dos tensiones primarias que se concentran en la división del trabajo del sistema de actividad. La primera tensión es la falta de una definición más clara y explícita de las funciones del profesor bilingüe. Conviene tener en cuenta que a diferencia de los otros dos dispositivos lingüísticos -aula de acogida y grupos interactivos-, la figura de facilitadores lingüísticos forma parte de las orientaciones y sugerencias que la política educativa ofrece a las escuelas (CHILE, 2017). Sin embargo, es un dispositivo que a la fecha cuenta con escasa atención de la investigación educativa y por tanto con pocas evidencias empíricas respecto a su funcionamiento (POBLETE, 2018). De nuestras observaciones participantes y posteriores entrevistas con docentes acompañados, se hace evidente que los profesores bilingües ingresan a aulas con el propósito general de ayudar a estudiantes con un bajo dominio de 
la lengua vehicular pero los propósitos más específicos no son evidentes ni a los ojos de un observador externo ni a los ojos del profesor que desarrolla la clase.

La segunda tensión, directamente relacionada con las anteriores, es la percepción de los docentes que la cantidad de facilitadores es limitada para la magnitud del apoyo necesario. Contar con uno o dos facilitadores por escuela cuando los estudiantes que requieren apoyo lingüístico pueden llegar a diez por aula, los posiciona como un recurso francamente exiguo y por tanto prácticamente inaccesible. La escasez y necesidad hace que el problema y las contradicciones emerjan a la hora de decidir con qué cursos, qué docentes o qué estudiantes se privilegiará el trabajo de estos profesionales.

De las tensiones presentadas, consideramos que las posibilidades de transformación expansiva de la escuela pasan por incrustar formalmente el dispositivo en el organigrama de trabajo de la unidad técnico-pedagógica, y de esta manera promover una mayor tipificación y jerarquización de las funciones docentes del equipo bilingüe. Finalmente, pensar en la incorporación de nuevos facilitadores pareciera ser una ucronía con lo cual las posibilidades de transformación van más en la línea de pensar formas más eficientes de utilizar los recursos/dispositivos, situación que podría darse, por ejemplo, a través de la implementación de nuevos artefactos mediadores, como es el caso del aula de acogida o bien de nuevas metodologías de aprendizaje como el caso de los grupos interactivos.

Con relación a la implementación del aula de acogida detectamos dos tensiones primarias que se concentran predominantemente en la comunidad del sistema de actividad. Recordemos que el aula de acogida es un espacio diseñado especialmente para el aprendizaje idiomático fuera del aula ordinaria. La principal tensión recae justamente en esta decisión, puesto que aun cuando los profesores valoran positivamente el aporte del aula de acogida, no deja de ser problemático el ser visto al mismo tiempo como un espacio de segregación educativa que no sólo erosiona el trabajo intercultural e inclusivo realizado por la escuela en otras dimensiones educativas, sino que interpela al proyecto educativo institucional en su conjunto. La pregunta que sintetiza esta tensión y que debiese ser respondida por la propia escuela es, ¿cómo responder a las necesidades de todos y todas las estudiantes a través de iniciativas inclusivas que eviten la segregación? En ese sentido el aula de acogida viene a reactivar las críticas a otros dispositivos por promover modelos educativos basados en simulacros de inclusión (VILLALOBOS-PARADA, 2014) o bien en repliegues relacionales (GONZÁLEZ, 2017).

La segunda tensión se ubica en torno a los múltiples puntos de vista, tradiciones e intereses que sobre los aprendizajes esperados existen al interior de la comunidad educativa. Como hemos descrito anteriormente, el aula de acogida es un dispositivo orientado al aprendizaje lingüístico, con lo cual no necesariamente guarda relación con los procesos de enseñanza-aprendizaje que se realizan en el aula ordinaria. La interrogante aquí es: ¿debe el aula de acogida contar con pertinencia curricular? Las voces están divididas, lo que ha significado que el equipo de gestión ha comenzado un proceso de revisión y potencial reformulación del dispositivo de manera a lograr una mayor vinculación entre el trabajo de aula ordinaria y el trabajo del aula de acogida. Al respecto, una de las principales críticas que ha recibido este dispositivo en países con una larga tradición migratoria, es justamente la artificialidad del trabajo realizado en comparación a la significatividad del aula ordinaria (GARCÍA, 2018), lo que redunda finalmente en una escasa incorporación de 
los profesionales a cargo de estas aulas al claustro de profesores. Ahora bien, la integración del aula de acogida al aula ordinaria está lejos de ser una solución simple y definitiva, puesto que implica activar otras tensiones en el ámbito de la división del trabajo del aula, ahora entre profesor de aula ordinaria y profesor de aula de acogida (DEL OLMO, 2012).

En cuanto a los grupos interactivos, estos se convierten en un dispositivo validado por el profesorado. Sin embargo, por sus características de sofisticación y secuencia procedimental, pueden correr el riesgo de centrarse más en la búsqueda de recursos didácticos que en la actividad como puente para generar aprendizajes en niños y niñas. A diferencia de los dispositivos anteriores, es en las normas y reglas de funcionamiento del sistema donde con mayor notoriedad se expresan tales complejidades. Al igual que otros programas de intervención orientados a la inclusión de estudiantes extranjeros como el modelo Quinta Dimensión (COLE; KOBELT; PACKER, 2014) o el programa Fondos de Conocimiento (ESTEBAN-GUITART; SERRA; LLOPART, 2018), los grupos interactivos pueden ser considerados en sí mismos un sistema de actividad (LAMAS; LALUEZA, 2016), con su propia división de trabajo, reglas y normas que organizan la comunidad de participantes. Esto es central y marca una distancia con los otros dispositivos, puesto que las principales tensiones y contradicciones se generan en su funcionamiento interno y no principalmente en el ensamblaje de éste con el sistema-escuela. Así, una de las principales tensiones de los grupos interactivos guarda relación con el reclutamiento de voluntarios que actúen como monitores de las estaciones de trabajo, situación que de acuerdo a nuestro trabajo de campo y reportado tanto por directores, docentes e incluso investigadores, se ha ido instalando como una dificultad estructural de los grupos interactivos. Si bien la propuesta original apuesta por el reclutamiento de familiares de los estudiantes o bien profesionales de las propias comunidades, lo cierto es que son un recurso inestable que amenaza su continuidad.

Podría plantearse la pregunta, ¿es posible un funcionamiento de grupos interactivos sin voluntarios? La respuesta ortodoxa es negativa puesto que impide garantizar uno de los propósitos centrales del dispositivo que es dinamizar las interacciones del grupo interactivo. Por lo tanto la contradicción está dada en la inflexibilidad de la norma que rige el funcionamiento del dispositivo para su buen funcionamiento. Adicionalmente, la necesaria formación/capacitación de los docentes en los principios educativos del programa -las actuaciones educativas de éxito- se constituye en una segunda tensión que limita las posibilidades de expandir los grupos interactivos hacia otros cursos o niveles educativos con agilidad. Aspectos como la distribución del tiempo, organización del espacio físico, diversificación del material de trabajo, individualización y ajuste de las ayudas ofrecidas, así como conocimiento en torno a diferentes enfoques de organización del aula y agrupamiento de estudiantes son las principales competencias requeridas para una adecuada implementación del dispositivo.

\section{Discusiones finales}

Como hemos corroborado en este trabajo, los dispositivos de acogida contienen en su propia naturaleza el potencial para resolver las contradicciones, tensiones y nudos críticos que surgen, generando ciclos de aprendizaje expansivo al interior de las escuelas. 
Es más, las propias escuelas cuentan con la agencia transformadora necesaria para promover estos ciclos, vale decir, con la capacidad de generar rupturas con relación a las medidas y actuaciones educativas tradicionales y la iniciativa para transformarlas (VIRKKUNEN; NEWNHAM, 2013; VIRKKUNEN et al., 2012). Una prueba de ello es el dispositivo de grupos interactivos, que incluye a estudiantes voluntarios como estrategia de modelación frente a nuevas soluciones ante actividades de aprendizaje (ENGESTRÖM, 2001). Esta implementación se encuentra en pleno proceso de desarrollo, y después de sus respectivos análisis, será posible determinar si tiene el mismo impacto que la modelación educativa desarrollada por el adulto.

Sin embargo, hay otras tensiones/contradicciones que permanecen vigentes. De esta forma y retomando las seis tensiones presentadas, consideramos que las posibilidades de transformación expansiva de la escuela se mueven en dos campos complementarios:

a) Por un lado una discusión necesaria que contribuya a encontrar puntos de encuentro entre la polifonía propia de la comunidad educativa respecto a qué modelo de gestión de la diversidad (GÓMEZ-HURTADO, 2013; JIMÉNEZ, 2014) será el que actúe como carta de navegación o hilo conductor de la escuela. A nuestro juicio, sin una claridad respecto a los enfoques, las contradicciones y tensiones internas saltarán constantemente como resortes en otras dimensiones del sistema de actividad, pudiendo dar origen a un formato de transformación reproductiva (ENGESTRÖM, 2009b). Señalábamos en la introducción de este trabajo la ausencia en Chile de una política educativa intercultural que aborde el fenómeno migratorio, quedando en manos de la política educativa inclusiva orientar el trabajo desarrollado por las comunidades (CHILE, 2017). Esto genera por ejemplo que uno de los dispositivos más prominentes a nuestro juicio como es el aula de acogida, puede ser valorado como una auténtica iniciativa inclusiva en tanto inhibe las barreras para el aprendizaje y la participación (CHILE, 2016), en este caso de estudiantes no castellanohablantes, puede ser al mismo tiempo valorado como una auténtica iniciativa de interculturalidad funcional (TUBINO, 2005), en tanto mantiene intactas las asimetrías de poder que caracterizan nuestro sistema educativo con relación a la presencia de minorías culturales. Esta que se expresa en lo local y en la cotidianeidad de una escuela determinada, se vive a su vez como una tensión no resuelta de la interculturalidad en nuestra región a nivel global (FERRÃ0, 2010). La tensión escolar no es otra cosa que una manifestación local de una discusión pendiente en torno a la educación intercultural latinoamericana.

b) Por otro lado una discusión necesaria en torno a la necesidad de abordar los fenómenos y problemáticas educativas desde una lógica de las inter-agencias relacionales entre profesionales (YAMAZUMI, 2009). Si asumimos el aprendizaje expansivo como un desplazamiento a través de la zona de desarrollo próximo del sistema-escuela que supone la incorporación de procesos de andamiaje (COLE; MEANS, 1986), la noción clásica de experticia debe ser deconstruida teniendo como referencia la metáfora de los tres cuartos (CHAIKLIN; HEDEGAARD, 2013). Esta metáfora sugiere que los agentes educativos no cuentan necesariamente con todos los conocimientos, habilidades o procedimientos necesarios para producir las transformaciones expansivas, puesto que siempre existen puntos ciegos imperceptibles para un agente individual. Por tanto, la completitud 
(cuarto restante), se obtiene trabajando conjuntamente con otros agentes, a través del establecimiento de agencias interprofesionales (YAMAZUMI, 2009). Como hemos constatado en trabajos anteriores (JIMÉNEZ et al., 2017; JIMÉNEZ; FARDELLA, 2015), han sido organizaciones no gubernamentales chilenas las que han contribuido formativamente con las comunidades educativas para avanzar en materia de interculturalidad en clave migratoria. Organismos como el Servicio Jesuita Migrante, Fundación para la Superación de la Pobreza, Fundación Educación 2020, Colectivo Sin Fronteras, Colectivo Trama, entre otros, ofrecen en la actualidad diferentes propuestas formativas en torno a educación intercultural. Algo similar ocurre en menor medida, desde el ámbito académico donde recientemente han sido publicadas guías pedagógicas de autoformación docente (FUNDACIÓN..., 2017).

Es en este escenario donde cobra relevancia que las políticas educativas en la materia vayan más allá de su histórico rol orientativo, presente en el caso de Chile en su Política Nacional de Estudiantes Extranjeros (CHILE, 2018b) y plasmado en la elaboración de orientaciones técnicas para la inclusión de estudiantes extranjeros (CHILE, 2017). En el marco de los resultados de esta investigación es que proponemos incorporar la figura de Equipos de Asesoramiento Escolar (EAS), vale decir, servicios educativos multidisciplinares que colaboren con las escuelas en la implementación de programas -o dispositivos específicos- de acogida. Somos conscientes que una figura de esta naturaleza supone desafiar estructuralmente la lógica de funcionamiento de las políticas educativas chilenas de neoliberalismo avanzado, caracterizadas por su inspiración en un modelo de mercado (FARDELLA; SISTO, 2015) y por la privatización y externalización de los servicios públicos (SISTO, 2019). Proponer por tanto la creación de equipos de asesoramiento escolar supone desafiar el funcionamiento circular instituido donde el Estado a través de su Ministerio de Educación prescribe, las comunidades educativas ejecutan dentro de sus posibilidades y finalmente el Estado a través de su Superintendencia de Educación y Agencia de Calidad fiscaliza y evalúa desempeños.

Una educación intercultural en clave migratoria no sólo debiese tener como horizonte la transformación de las instituciones escolares, el empoderamiento de las minorías culturales que forman parte de ellas o la descolonización de las epistemologías a la base de éstas (DIETZ; MATEOS, 2013), sino principalmente tensionar y transformar los modelos educativos en los que dicha educación intercultural se inscribe. Una educación intercultural que transite desde su estado actual indigenista y con enfoque racialcompensatorio (VILLALTA, 2016), hacia un nuevo horizonte interseccional (SÁNCHEZMELER0; GIL-JAURENA, 2015) y emancipatorio.

\section{Referencias}

ALEGRE, Miguel; BENITO, Ricard; GONZÁLEZ, Sheila. Experiencias escolares iniciales del alumnado inmigrado: comienzos que marcan. Educación XXI, Madrid, v. 15, n. 2, p. 137-158, 2012.

BRAV0, Ricardo. Inmigrantes en la escuela chilena: ciertas representaciones para ciertas políticas en educación. Revista Latinoamericana de Educación Inclusiva, Santiago de Chile, v. 6, n. 1, p. 39-52, 2012. 
CAMPOS-BUSTOS, Juana. Estudiantado haitiano en Chile: aproximaciones a los procesos de integración lingüística en el aula. Revista de Educación, San José, v. 43, n. 1, p. 1-17, 2019. D0l: <https://doi. org/10.15517/revedu.v43i1.30458>.

CHAIKLIN, Seth; HEDEGAARD, Mariane. Cultural-historical theory and educational practice: some radicallocal considerations. Nuances, São Paulo, v. 24, n .1, p. 30-44, 2013.

CHILE. Ministerio de Educación. Orientaciones para la construcción de comunidades educativas inclusivas. Santiago de Chile: Ministerio de Educación: Gobierno de Chile, 2016.

CHILE. Ministerio de Educación. Orientaciones - Programa de Educación Intercultural Bilingüe. Santiago de Chile: Ministerio de Educación: Gobierno de Chile, 2005.

CHILE. Ministerio de Educación. Orientaciones técnicas para la inclusión educativa de estudiantes extranjeros. Santiago de Chile: Ministerio de Educación: Gobierno de Chile, 2017.

CHILE. Ministerio de Educación. Política nacional de estudiantes extranjeros 2018-2022. Santiago de Chile: Ministerio de Educación: Gobierno de Chile, 2018b.

CHILE. Ministerio de Educación. Programa de Educación Intercultural Bilingüe 2010-2016. Santiago de Chile: Ministerio de Educación: Gobierno de Chile, 2018a.

COLE, Michael; KOBELT, Elías; PACKER, Martin. La metodología de la investigación para una psicología concreta: la investigación con la Quinta Dimensión. Psicología, Conocimiento y Sociedad, Montevideo, v. 4, n. 2, p. 28-61, 2014.

COLE, Michael; MEANS, Bárbara. Cognición y pensamiento. Buenos Aires: Paidós, 1986.

CRENSHAW, Kimberlé. From private violence to mass incarceration: thinking intersectional about women, race and social control. UCLA Law Review, Los Ángeles, v. 59, p. 1418-1472, 2012.

DEL OLMO, Margarita. Buenas prácticas, ¿desde el punto de vista de quién? Una contribución a la controversia sobre las aulas de enlace. Revista de Educación, Madrid, v. 358, p. 111-128, 2012.

DÍAZ DE RADA, Ángel. Etnografía de la escuela más allá de la etnografía y de la escuela: tensiones disciplinares y aplicabilidad de los saberes etnográficos. Educación y Futuro, Madrid, n. 29, p. 13-39, 2013.

DIETZ, Gunther; MATEOS, Laura. Interculturalidad y educación intercultural en México: un análisis de los discursos nacionales e internacionales en su impacto en los modelos educativos mexicanos. México: Coordinación General de Educación Intercultural y Bilingüe, 2013.

DIEZ, María. Reflexiones en torno a la interculturalidad. Cuadernos de Antropología Social, Buenos Aires, v. 19, p. 191-213, 2004.

ELIGE EDUCAR. Un nuevo desafío para la educación chilena: ser un país de migrantes. Aula maestra 10. Santiago de Chile: Colección Servicios Educativos, 2017. Cambridge: Cambridge University Press, 2009a. p. 303-328. 
ENGESTRÖM, Yrjö. El aprendizaje expansivo en el trabajo: hacia una reconceptualización teórica de la actividad. Journal of Education and Work, Bath, v. 14, n. 1, p. 1-16, 2001.

ENGESTRÖM, Yrjö. Enriching the theory of expansive learning: lessons from journeys toward coconfiguration. Mind, Culture, and Activity, Copenhague, v. 14, n. 1/2, p. 23-39, 2007.

ENGESTRÖM, Yrjö. From learning environments and implementation to activity systems and expansive learning. Actio, Helsinki, n. 2, p. 17-33, 2009b.

ENGESTRÖM, Yrjö. Learning by Expanding. Cambrigde: Cambridge University Press, 1987.

ENGESTRÖM, Yrjö. The future of activity theory: a rough draft. In: SANNINO, Annalisa; DANIELS, Harry; GUTIÉRREZ, Kris (Ed.). Learning and expanding with activity theory. Cambrigde: Cambridge University Press, 2009a.

ERAUSQUIN, Cristina. La teoría histórico-cultural de la actividad como artefacto mediador para construir intervenciones e indagaciones sobre el trabajo de psicólogos en escenarios educativos. Revista de Psicología, Ciudad de la Plata, v. 13, p. 173-197, 2014.

ESSOMBA, Miquel. Inmigración, sociedad y educación en la UE: hacia una política educativa de plena inclusión. Cultura y Educación, Madrid, v. 24, n. 2, p. 137-148, 2012.

ESTEBAN-GUITART, Moisés; SERRA, Josep; LLOPART, Mariona. The role of the study group in the funds of knowledge approach. Mind, Culture, and Activity, Copenhague, v. 25, n. 3, p. 216-228, 2018.

FARDELLA, Carla; SISTO, Vicente. Nuevas regulaciones del trabajo docente en Chile: discurso, subjetividad y resistencia. Psicologia \& Sociedade, Recife, v. 2, n. 1, p. 68-79, 2015.

FERNÁNDEZ, José; GARCÍA CASTAÑO, Francisco. El desarrollo normativo que regula las aulas escolares de nacionalidad extranjera. Profesorado, Granada, v. 19, n. 1, p. 468-495, 2015.

FERRÃO, Vera. Educación intercultural en América Latina: distintas concepciones y tensiones actuales. Estudios Pedagógicos, Valdivia, v. 36, n. 2, p. 333-342, 2010.

FLICK, Urie. An introduction to qualitative reserch. Limited: Sage, 2014.

FUNDACIÓN SUPERACIÓN DE LA POBREZA. Educación e interculturalidad en escuelas públicas: orientaciones desde la práctica. Santiago de Chile: FSP, 2017.

GARCÍA, Raúl. Análisis de las aulas de acogida lingüística: carácter inclusivo, flexibilidad e integración en la organización del centro. Tendencias Pedagógicas, Madrid, v. 32, p. 91-105, 2018.

GARRETA, Jordi. Ethnic minorities in the Spanish and Catalan educational systems: from exclusion to intercultural education. International Journal of Intercultural Relations, Coral Gables, v. 30, n. 2, p. 261-79, 2006.

GOMEZ-HURTADO, Inmaculada. Dirección y gestión de la diversidad en la escuela: hacia un liderazgo inclusivo. Revista Fuentes, Sevilla, n. 14, p. 61-84, 2013. 
GONZÁLEZ, Sheila. Las aulas de acogida, la procedencia y la lengua: ¿factores de repliegue relacional en los institutos? Interacções, Lisboa, v. 13, n. 43, p. 179-204, 2017.

GONZALEZ-FALCON, Inmaculada; MOYA, Asunción; DUSI, Paola. La inclusión del alumnado de origen extranjero en los centros de educación secundaria de Andalucía: una aproximación cualitativa desde el aula temporal de aceleración lingüística. Gazeta de Antropología, Granada, v. 34, n. 1, p. 1-17, 2018.

JIMÉNEZ, Felipe. Modelos de gestión de la diversidad cultural para la escolarización de alumnado inmigrante en las escuelas chilenas: desafíos para la interculturalidad actual. Estudios Pedagógicos, Valdivia, v. 40, n. 2, p. 409-426, 2014.

JIMÉNEZ, Felipe; FARDELLA, Carla. Diversidad y rol de la escuela: discursos del profesorado en contextos educativos multiculturales en clave migratoria. Revista Mexicana de Investigación Educativa, Ciudad de México, v. 20, n. 65, p. 419-44, 2015.

JIMÉNEZ, Felipe; VALDÉS, René; AGUILERA, María. Geografías de la investigación académica sobre migración y escuela: voces, silencios y prospectivas de nuestra profesión. Estudios Pedagógicos, Valdivia, v. 34, n. 3, p. 173-191, 2018.

JIMÉNEZ, Felipe et al. Migración y escuela: análisis documental en torno a la incorporación de inmigrantes al sistema educativo chileno. Psicoperspectivas, Viña del Mar, v. 16, n. 1, p. 105-116, 2017.

KONTOS, Cynthia. Teoría de la actividad. Aprendizaje expansivo e instrumentos para la reflexión sobre la práctica profesional, interacción alumno-docente y alumno-alumnos, y otros agentes en el aula taller. Escritos en la Universidad, Buenos Aires, n. 124, p. 67-69, 2016.

LAMAS, Macarena; LALUEZA, José. Innovar en el aula. Contradicciones entre nuevas herramientas y viejos roles como medio para trasformar la práctica. Estudios Pedagógicos, Valdivia, v. 42, n. 3, p. 243-258, 2016.

LARRIPA, Martín; ERAUSQUIN, Cristina. Teoría de la actividad y modelos mentales. Instrumentos para la reflexión sobre la práctica profesional: «aprendizaje expansivo», intercambio cognitivo y transformación de intervenciones de psicólogos y otros agentes en escenarios educativos. Anuario de Investigaciones, Buenos Aires, v. 15, p. 109-124, 2008.

MAGLIANO, María. Interseccionalidad y migraciones: potencialidades y desafíos. Revista Estudos Feministas, Santa Catarina, v. 23, n. 3, p. 691-712, 2015.

MONTECINOS, Carmen. Analizando la política de educación intercultural bilingüe en Chile desde la educación multicultural. Cuadernos Interculturales, Valparaíso, v. 2, n. 3, p. 25-32, 2004.

MORA, María. Política educativa para migrantes en Chile: un silencio elocuente. Polis, Santiago de Chile, v. 17, n. 49, p. 231-257, 2018.

OLMOS, Antonia; LASTRES, Nazaret. Transitando por los bordes de la integración: una aproximación etnográfica a políticas educativas y experiencias escolares de alumnado que desconoce la lengua vehicular de la escuela en Andalucía (España). Gazeta de Antropología, Granada, v. 34, n. 1, artículo 4, 2018. 
OLMOS, Antonia; RUBI0, María. Imaginarios sociales sobre "la/el buen y la/el mal estudiante": sobre la necesidad de un análisis interseccional para entender las lógicas de construcción de la diferencia hacia el alumnado "inmigrante". In: CUCALÓN, Pilar (Ed.). Etnografía de la escuela y la Interseccionalidad. Madrid: Traficante de Sueños, 2014. p. 9-15.

PALAUDÀRIAS, Josep; GARRETA, Jordi. La acogida del alumnado de origen inmigrante: un análisis comparado desde la situación en Cataluña. Revista Española de Educación Comparada, Madrid, n. 14, p. 49-78, 2008.

POBLETE, Rolando. El trabajo con la diversidad desde el currículo en escuelas con presencia de niños y niñas migrantes: estudio de casos en escuelas de Santiago de Chile. Perfiles Educativos, Ciudad de México, v. 40, n. 159 , p. 51-65, 2018.

POBLETE, Rolando; GALAZ, Caterine. Aperturas y cierres para la inclusión educativa de niños/as migrantes en Chile. Estudios Pedagógicos, Valdivia, v. 43, n. 3, p. 239-257, 2017.

RIEDEMANN, Andrea. Alumnos inmigrantes en escuelas chilenas: una mirada a las nuevas desigualdades del sistema educativo. In: VERA, Antonieta (Ed.). Malestar social y desigualdades en Chile. Santiago de Chile: Universidad Alberto Hurtado, 2017. p. 99-121.

RIEDEMANN, Andrea. La educación intercultural bilingüe en Chile: ¿ampliación de oportunidades para alumnos indígenas? Indiana, Berlín, v. 25, p. 169-193, 2008.

SANCHEZ-MELERO, Hector; GIL-JAURENA, Inés. Análisis interseccional y enfoque intercultural en el estudio de la ciudadanía y la participación: consideraciones epistemológicas. Diálogo Andino, Arica, n. 47, p. 143149, 2015.

SANNINO, Annalisa; ENGESTRÖM, Yrjö; LEMOS, Mónica. Formative interventions for expansive learning and transformative agency. Journal of the Learning Sciences, Philadelphia, v. 25, n. 4, p. 599-633, 2016.

SIMÓ, Nuria et al. La acogida educativa en los centros escolares en Cataluña: más allá de los recursos específicos para el alumnado de nueva incorporación. Revista Complutense de Educación, Madrid, v. 25, n. 1, p. 177-194, 2014

SISTO, Vicente. Inclusión "a la Chilena": la inclusión escolar en un contexto de políticas neoliberales avanzadas. Archivos Analíticos de Políticas Educativas, Arizona, v. 27, n. 23, p. 1-21, 2019.

STEFONI, Carolina; STANG, Fernanda; RIEDEMANN, Andrea. Educación e interculturalidad en Chile: un marco para el análisis. Estudios Internacionales, Santiago de Chile, v. 48, n. 185, p. 153-182, 2016.

SUMONTE, Valeria et al. Migración no hispanoparlante en chile: tendiendo puentes lingüísticos e interculturales. Diálogo Andino, Arica, n. 57, p. 57-67, 2018.

TUBINO, Fidel. La interculturalidad crítica como proyecto ético-político. In: ENCUENTRO CONTINENTAL DE EDUCADORES AGUSTINOS, 2005, Lima. Actas... Lima: [s. n.], 2005. p. 1-4. Disponible en: <http://oala. villanova.edu/congresos/educación/lima-ponen-02.html>. Acceso en: 7 mzo. 2018. 
VALDÉS, René et al. Dispositivos de acogida a estudiantes extranjeros como plataformas de intervención formativa. Estudios Pedagógicos, Valdivia, v. 45, n. 2, p. 1-17, 2019.

VÁZQUEZ, Félix. El análisis de contenido temático: objetivos y medios en la investigación psicosocial. Documento de Trabajo, Barcelona, 1996, p. 47-70.

VILLALOBOS-PARADA, Boris. Simulacro de inclusión: normalización y Convivencia Escolar. In: CONGRESO INTERNACIONAL SOBRE VIOLENCIA EN LAS ESCUELAS, 3., 2014, Buenos Aires. Congreso... Buenos Aires: [s. n.], 2014. p. 1-8. Comunicación presentada.

VILLALTA, Marco. Educación intercultural en Latinoamérica: análisis de las investigaciones de campo en la región. Psicoperspectivas, Viña del Mar, v. 15, n. 1, p. 130-146, 2016.

VIRKKUNEN, Jaakko; NEWNHAM, Denise. The change laboratory: a tool for collaborative development of work and education. Rotterdam: Sense, 2013.

VIRKKUNEN, Jaakko et al. Breaking the vicious circle of categorizing students in school. Learning, Culture and Social Interaction, Oxford, v. 66, p. 183-192, 2012.

VYGOTSKY, Lev. Pensamiento y lenguaje. Cambridge: MIT Press, 1986.

WALSH, Catherine. Interculturalidad crítica y educación intercultural. In: SEMINARIO INTERNACIONAL DE INVESTIGACIÓN EDUCATIVA, INTERCULTURALIDAD Y EDUCACIÓN INTERCULTURAL, 2., 2009, La Paz. Segundo... La Paz: Bogotá: Editorial III-CAB, 2009. p. 1-18.

YAMAZUMI, Katsuhiro. Expansive agency in multi-activity collaboration. In: SANNINO, Annalisa; DANIELS, Harry; GUTIÉRREZ, Kris (Ed.). Learning and expanding with activity theory. Cambridge: Cambridge University Press, 2009. p. 212-227.

Recibido en: 21.01.2019

Revisado en: 21.05 .2019

Aprobado en: 14.08.2019

Felipe Jiménez-Vargas es doctor en Psicología de la Educación por la Universidad Autónoma de Barcelona, España. Profesor asociado de la Escuela de Psicología de la Pontificia Universidad Católica de Valparaíso. Sus líneas de investigación son migración y educación intercultural.

René Valdés Morales es doctor en Psicología por la Pontificia Universidad Católica de Valparaíso. Es profesor agregado de la Escuela de Pedagogía de la misma universidad e investigador postdoctorante (Conicyt) por la Universidad Andrés Bello. Sus líneas de investigación son liderazgo escolar e inclusión educativa. 
María-Teresa Hernández-Yáñez es doctora en Ciencias de la Educación por la Universidad de Santiago de Chile y magíster en Educación. Es educadora de párvulos. Sus líneas de investigación son Infancia y Educación intercultural.

Carla Fardella es doctora en Psicología Social por la Universidad Autónoma de Barcelona, España. Es investigadora asociada de la Facultad de Educación y Ciencias Sociales de la Universidad Andrés Bello. Sus líneas de investigación son análisis de políticas públicas y estudios sociales del trabajo. 
SECCIÓN: ERRATA

Errata: http://dx.doi.org/10.1590/s1517-9702202046218867E

En el artículo "Dispositivos lingüísticos de acogida, aprendizaje expansivo e interculturalidad: contribuciones para la inclusión educativa de estudiantes extranjeros" http://dx.doi.org/10.1590/s1678-4634202046218867 publicado en Educação e Pesquisa, v. 46, p. 01-21, e-location e218867, 2020.

Página 03, segundo párrafo.

Donde se leyó a Ahora bien y pese al aumento de investigaciones en torno al fenómeno migratorio en nuestro sistema educativo, aumento que hemos podido mapear en una reciente revisión (JIMÉNEZ; VALDÉZ; HERNÁNDEZ, 2018),...

Leer Ahora bien y pese al aumento de investigaciones en torno al fenómeno migratorio en nuestro sistema educativo, aumento que hemos podido mapear en una reciente revisión (JIMÉNEZ; VALDÉS; AGUILERA, 2018),...

Página 05, final del cuarto párrafo.

Donde se leyó a En el caso de Chile y como hemos podido documentar en investigaciones previas, la mayoría de los dispositivos de acogida se condensan en la dimensión administrativa y en la dimensión convivencial de los procesos educativos (JIMÉNEZ; VALDÉZ; HERNÁNDEZ, 2018).

Leer En el caso de Chile y como hemos podido documentar en investigaciones previas, la mayoría de los dispositivos de acogida se condensan en la dimensión administrativa y en la dimensión convivencial de los procesos educativos (JIMÉNEZ; VALDÉS; AGUILERA, 2018).

Página 18,

Donde se leyó a JIMÉNEZ, Felipe; VALDÉS, René; HERNÁNDEZ, María. Geografías de la investigación académica sobre migración y escuela: voces, silencios y prospectivas de nuestra profesión. Estudios Pedagógicos, Valdivia, v. 34, n. 3, p. 173-191, 2018.

Leer JIMÉNEZ, Felipe; VALDÉS, René; AGUILERA, María. Geografías de la investigación académica sobre migración y escuela: voces, silencios y prospectivas de nuestra profesión. Estudios Pedagógicos, Valdivia, v. 34, n. 3, p. 173-191, 2018. 